EPJ manuscript No.

(will be inserted by the editor)

\title{
Synchronizability of chaotic logistic maps in delayed complex networks
}

\author{
Marcelo Ponce C. ${ }^{1}$, C. Masoller ${ }^{2}$ and Arturo C. Martí ${ }^{1}$ \\ 1 Instituto de Física, Facultad de Ciencias, Universidad de la República, Iguá 4225, Montevideo 11400, Uruguay \\ 2 Departament de Fisica i Enginyeria Nuclear, Universitat Politecnica de Catalunya, Colom 11, E-08222 Terrassa, Barcelona, \\ Spain
}

July 25, 2018

\begin{abstract}
We study a network of coupled logistic maps whose interactions occur with a certain distribution of delay times. The local dynamics is chaotic in the absence of coupling and thus the network is a paradigm of a complex system. There are two regimes of synchronization, depending on the distribution of delays: when the delays are sufficiently heterogeneous the network synchronizes on a steady-state (that is unstable for the uncoupled maps); when the delays are homogeneous, it synchronizes in a time-dependent state (that is either periodic or chaotic). Using two global indicators we quantify the synchronizability on the two regimes, focusing on the roles of the network connectivity and the topology. The connectivity is measured in terms of the average number of links per node, and we consider various topologies (scale-free, small-world, star, and nearest-neighbor with and without a central hub). With weak connectivity and weak coupling strength, the network displays an irregular oscillatory dynamics that is largely independent of the topology and of the delay distribution. With heterogeneous delays, we find a threshold connectivity level below which the network does not synchronize, regardless of the network size. This minimum average number of neighbors seems to be independent of the delay distribution. We also analyze the effect of selffeedback loops and find that they have an impact on the synchronizability of small networks with large coupling strengths. The influence of feedback, enhancing or degrading synchronization, depends on the topology and on the distribution of delays.
\end{abstract}

PACS. 0 5.45.Xt, 05.45.-a, 05.45.Ra, 05.45.Pq

\section{Introduction}

Complex networks arise due to self-organization phenomena in many real systems, such as food webs, the Internet, social networks, genes, cells and neurons 1. A lot of research has been devoted to understanding the collective behavior emerging in complex networks [2, given the individual dynamics of the nodes and the coupling architecture. Ecological webs, for example, describe species by means of nodes connected by links, representing the interactions. The interactions can be either direct or indirect through intermediate species, and can be of antagonistic type, such as predation, parasitism, etc., or mutually beneficial, such as those involving the pollination of flowers by insects $[3$. A study of the network architecture decompose food webs in spanning trees and loop-forming links, revealing common principles underlying the organization of different ecosystems [4]. Cells use metabolic networks of interacting molecular components in processes that generate mass, energy, information transfer and cell-fate specification [5]. Adaptation and robustness have been shown to be consequences of the network's connectivity and do not require the 'fine-tuning' of parameters [6]. In a cat's brain, functional connectivity has been studied within the framework of complex networks [7]. In the human brain, magnetic resonance imaging has been used to extract functional networks connecting correlated human brain sites [8]. Analysis of the resulting networks in various tasks (e.g., move a finger) has shown interesting features in the brain, such as scale-free structure, a high clustering coefficient, and a small characteristic path length.

Complex network are relevant not only from an academical point of view but also from an applied perspective. Models based on complex networks for the spread of diseases have identified mitigation strategies for epidemic spread. In [9] the spread of an infection was analyzed for different population structures, ranging from ordered lattices to random graphs, and it was shown that for the more ordered structures, there was a fluctuating endemic state of low infection. In [10] it was shown that outbreaks can be contained by a strategy of targeted vaccination combined with early detection, thus avoiding mass vaccination of the hole population. In communication networks, error tolerance and attack vulnerability are key issues. In [1] it was shown that error tolerance is not shared by all networks 
Marcelo Ponce C., C. Masoller, Arturo C. Martí: Synchronizability of chaotic logistic maps...

that contain redundant wiring, but is displayed only by those with scale-free topology.

The structure of a network is a key issue in determining its functional properties. In real networks communities, or modules, associated with highly interconnected parts, have been identified [12,13. A nice example of a community structure has been unveil in networks of musical tastes [14, having also practical applications for the development of commercial music recommendation engines.

Well-characterized modules have been identified in synthetic gene networks [15, where positive feedback and noise play important roles for the repression and the activation of gene expression. An excitable module containing positive and negative feedback loops has been identified as a key mechanism inducing transient cellular differentiation [16. A method for classifying nodes into universal roles according to their intra- and inter-module connections has been recently proposed [17] and applied to the study metabolic networks.

The synchronizability of a network, or is propensity for synchronization, is another key issue in determining the network functional properties [18]. Why some topologies are easier to synchronize than others, is still an issue not fully understood. Heterogeneity in the connection strengths tends to enhance synchronization [19]. A weighting procedure based upon the global structure of network pathways has been shown to improve synchronizability 20. Dynamical adaptation, where the coupling strengths develop according to the local synchronization of the node and its neighbors, resulting in weighted coupling strengths that are correlated with the topology, also enhances synchronizability [21. However, heterogeneity in the connectivity distribution can have the opposite effect: networks with homogeneous connectivity have been found to have larger propensity for synchronization than the heterogeneous ones 22].

The speed of transmission of information among the network also affects the synchronizability. Instantaneous interactions have been studied a lot in spite of the fact in many situations they are not realistic, because the information propagates with a finite speed. A more realistic scenario considers that the links have associated delay times, and that the delays are the same for all the links. It has been shown that the presence of such uniform delays in the communications among the nodes can result in enhanced synchronizability. In 23 it was shown that, in a network of chaotic maps, it was possible to synchronize the delayed network where the undelayed network, with instantaneous links, did not.

An even more realistic approach for complex and disordered systems is to consider heterogeneously distributed delays. In previous work 24,25] we studied networks of coupled maps with links that have heterogenous delays, and investigated the relation of the network topology with its ability to synchronize. We found that (i) the synchronizability was enhanced by random delays as compared to networks with uniform delays, (ii) the network synchronized in a steady state in the presence of random delays (in contrast, with uniform delays the synchronization is in time a dependent state, 23) and (iii) the synchronizability depends mainly on the mean connectivity and is rather independent of the topology.

The aim of this paper is to further analyze how the synchronizability depends on the connectivity and on the topology, when there are delays in the links among the nodes. We consider both regular and random network topologies, covering the cases of homogeneous and heterogeneous distribution of the links. We also study the effects of centrality and locality on the dynamics of the array. We characterize the synchronizability in terms of two indicators, one that tends to zero when the networks synchronizes, regardless if the synchronization is in a time dependent or in a steady state, and the other that tends to zero only when the synchronization is in a steady state. Using these indicators we also analyze the impact of self-feedback links. This paper is organized as follows. Section 2 presents the model, the different distributions of delays, and several topologies used. Section 3 presents the results of the simulations, and, finally, Sec. 4 presents a summary and the conclusions.

\section{Model}

We consider $N$ logistic maps coupled as:

$$
x_{i}(t+1)=(1-\epsilon) f\left[x_{i}(t)\right]+\frac{\epsilon}{b_{i}} \sum_{j=1}^{N} \eta_{i j} f\left[x_{j}\left(t-\tau_{i j}\right)\right],
$$

where $t$ is a discrete time index, $i$ is a discrete spatial index $(i=1 \ldots N), f(x)=a x(1-x)$ is the logistic map, $\epsilon$ is the coupling strength and $\tau_{i j} \geq 0$ is the delay time in the interaction between the $i$ th and $j$ th nodes (the delay times $\tau_{i j}$ and $\tau_{j i}$ need not be equal). The matrix $\eta=\left(\eta_{i j}\right)$ defines the connectivity of the network: $\eta_{i j}=\eta_{j i}=1$ if there is a link between the $i$ th and $j$ th nodes, and zero otherwise. The sum in Eq. (1) runs over the $b_{i}$ nodes which are coupled to the $i$ th node, $b_{i}=\sum_{j} \eta_{i j}$. The normalized pre-factor $1 / b_{i}$ means that each map receives the same total input from its neighbors.

A particularly simple solution of Eq. (1) is such that all the maps of the network are in a fixed point of the uncoupled map, i.e.,

$$
x_{i}(t)=x_{0} \quad \forall \quad i,
$$

with $x_{0}=f\left(x_{0}\right)$. While this solution exists for all delay distributions $\left(\tau_{i j}\right)$ and for all coupling topologies $\left(\eta_{i j}\right)$, the statistical linear stability analysis performed in [24] showed that this solution is unstable unless the distribution of delays is wide enough. In the other limiting case of all-equal delays, $\tau_{i j}=\tau \forall i$ and $j$, it was shown in Ref. 23. that the network synchronizes isochronally, in a spatially homogeneous time-dependent state:

$$
x_{i}(t)=x(t) \quad \forall \quad i,
$$

with $x(t+1)=(1-\epsilon) f[x(t)]+\epsilon f[x(t-\tau)]$. 


\subsection{Distribution of delays}

We consider delays distributed as: $\tau_{i j}=\tau_{0}+\operatorname{near}(c \xi)$, where $c$ is a parameter that allows varying the width of the delay distribution; $\xi$ is Gaussian distributed with zero mean and standard deviation one; near denotes the nearest integer. Depending on $\tau_{0}$ and $c$ the distribution of delays is truncated to avoid negative delays.

The synchonizability of the network depends on both, the mean delay and the width of the delay distribution [24]. The larger the value of $\tau_{0}$, the larger the dispersion of the delays has to be, for the network to synchronize in the steady state (see Fig. 3 of [24]). A similar observation was recently reported in 32, for an integro-differential equation describing the collective dynamics of a neural network with distributed signal delays. An interesting interpretation of both observations is provided by the work of Morgado et al. 33]: a certain degree of randomness in a network (due to random delays, random connectivity, or even random initial conditions) results in additive and/or multiplicative noise terms in an "effective" singlenode equation of motion for $x_{i}(t+1)$, expressed in terms of $x_{i}(t)$ and nonlinear feedback memory terms, that for the Logistic map are of polynomial type in $x_{i}(t-n)$ with $1<n<t$. In [33] it was found that the right memory profile can create optimal conditions for synchronization, in other words, an optimal memory range enhances a network propensity for synchronization.

Since our aim is to study the roles of the topology and of the connectivity, we keep fixed the distribution of delays, defined by the parameters $\tau_{0}$ and $c$. However, we compare the results obtained for distributed delays $(c \neq 0)$ with those obtained for all-equal delays $(c=0)$ and with those obtained for no delays $\left(\tau_{0}=0, c=0\right)$.

\subsection{Network connectivity and topology}

The connectivity of a network is measured in terms of the average number of links per node,

$$
\langle b\rangle=\frac{1}{N} \sum_{i=1}^{N} b_{i} .
$$

We consider the five topologies, three of them are regular networks where the links are distributed deterministically among the nodes, while the other two are heterogenous networks where the links are distributed stochastically, with given rules. The networks are:

(i) a nearest-neighbor network (referred to as NN network) with periodic boundary conditions where each node $i$ is linked to its neighboring nodes $i \pm 1, i \pm 2, \ldots, i \pm K$, with $K$ an integer. The number of neighbors is the same for all the nodes, and $\langle b\rangle=2 K$.

(ii) the NN network with the addition of a central node connected to all other nodes (referred to as ST network). In this case, $N-1$ nodes have $2 K+1$ links and one node has $N-1$ links. Thus, $\langle b\rangle=[(2 K+1)(N-1)+(N-1)] / N=$ $(2 K+2)(N-1) / N$. (iii) a star-type network where there are $K$ central nodes that are connected to all other nodes and $N-K$ peripheric nodes that are connected only to the central ones (referred to as KA). In this case, $K$ nodes have $N-1$ links and $N-K$ nodes have $K$ links. Thus, $\langle b\rangle=$ $[K(N-1)+(N-K) K] / N$. This network has a pure startype structure and is centrally organized, in contrast to NN, that is locally organized.

(iv) a small-world (SW) network constructed according to the Newman and Watts algorithm [26].

(v) a scale-free (SF) network constructed according to the Barabasi and Albert method [27].

Recently, the influence of feedback loops on the synchronization of complex systems has received attention. In networks of globally coupled units (self-sustained oscillators or maps) delayed feedback in the mean field can, depending on the feedback strength and on the delay time, enhance or suppress synchronization 28. In a system composed by two phase oscillators with instantaneous mutual coupling, a delayed feedback loop in each oscillator enhances synchronizability if the coupling strength is not too strong, while degrades synchronizability if the coupling exceeds a threshold value 29. In small networks of mutually delayed-coupled oscillators, which typically do not exhibit stable isochronal synchronization, by including delayed feedback loops to the nodes, the oscillators become isochronally synchronized 30,31. To analyze the relevance of feedback loops we consider two situations: the diagonal elements of the coupling matrix, $\eta_{i i}$, are all set equal to 1 , or are all set equal to 0 . In this way, in each node a feedback loop is included, or is forbidden. The delays of the feedback loops, $\tau_{i i}$, have the same distribution as the delays of the mutual interactions, $\tau_{i j}$.

\subsection{Synchronization indicators}

To characterize the degree of synchronization and to distinguish between steady state and time dependent synchronization, we use the following indicators,

$$
\begin{aligned}
\sigma^{2} & =\frac{1}{N}\left\langle\sum_{i}\left(x_{i}-\langle x\rangle_{s}\right)^{2}\right\rangle_{t} \\
\sigma^{\prime 2} & =\frac{1}{N}\left\langle\sum_{i}\left(x_{i}-x_{0}\right)^{2}\right\rangle_{t},
\end{aligned}
$$

where $\langle.\rangle_{s}$ denotes a space average over the nodes of the network, $\langle.\rangle_{t}$ denotes a time average, and $x_{0}$ is the fixed point of the uncoupled logistic map, $x_{0}=f\left(x_{0}\right) \cdot \sigma^{2}=0$ if and only if $x_{i}=x_{j} \forall i, j$, while $\sigma^{\prime 2}=0$ if and only if $x_{i}=x_{0} \forall i$. Thus, $\sigma^{\prime 2}$ allows to distinguish synchronization in the steady state from synchronization in an time dependent state. In the former case, both $\sigma^{2}$ and $\sigma^{\prime 2}$ are zero, in the latter case, only $\sigma^{2}=0$.

\section{Results}

In this section we present the results of the simulations. We consider a network of $N=200$ logistic maps with 
$a=4$ interacting with different topologies, as described above. The parameters of the Gaussian delay distribution are $\tau_{0}=5$ and $c=2$. Similar results are found for other values of $\tau_{0}$ and $c$, with $c$ large enough 24 . To asses the role of distributed delays, we compare with two limits: (i) instantaneous coupling, $\tau_{i j}=0 \forall i, j$, and (ii) homogeneous delays, $\tau_{i j}=\tau_{0} \forall i, j(c=0)$.

The simulations start from a random initial configuration, with $x_{i}(0)$ randomly distributed in $[0,1]$, and the maps evolve initially without coupling, during a time interval $0<t<\max \left(\tau_{i j}\right)$, because the integration of delayed equations requires the knowledge of the past state of the system over a time interval given by the maximum delay. After that, the coupling is turned on. We neglect transient effects disregarding a few thousands of iterations.

Networks of coupled elements usually show multistability: different initial conditions lead to different final states, and delayed coupling tends to increase the number of coexisting states [34,35]. Multistability is also enhanced when the local dynamics of the uncoupled maps presents two or more competing attractors [36, which does not occur for the logistic map with $a=4$. When the coupling is weak and the network is not synchronized, we observe a large diversity of dynamical clustered states. However, in this "weak coupling regime" the global synchronization indicators, $\sigma^{2}$ and $\sigma^{\prime 2}$, depend mainly on $\epsilon$ and only in a small extend on the initial conditions, the distribution of delays, the network topology and the connectivity (with the exception of all-even delays, for them there is a synchronization "island" that will be discussed below). In the following, the plots of $\sigma^{2}$ and $\sigma^{\prime 2}$ are done by averaging over 10 different states generated from randomly chosen initial conditions. The only exception is Fig. 6, that is done with just one initial condition (that is the same for all $\epsilon$ ).

In the first section we characterize the network propensity for synchronization in terms of the indicator $\sigma^{2}$; in the second section, in terms of $\sigma^{\prime 2}$. In these two sections the network does not contain self-feedback links $\left(\eta_{i i}=0 \forall i\right)$. In the last section we asses the role of feedback links, by studying the same networks (with the same delay distributions and topologies) but with a feedback link in each node $\left(\eta_{i i}=1 \forall i\right)$.

\subsection{Characterization in terms of $\sigma^{2}$}

Figures 1 and 2 display color-coded plots of the synchronization indicator $\sigma^{2}$ as a function of the coupling strength, $\epsilon$, and the average number of neighbors, $\langle b\rangle$, for the different topologies and delay distributions.

Figure 1 displays results for the regular topologies (NN: top row, ST: central row, KA: bottom row) and the three distributions of delays considered (no-delays: left column, homogeneous delays; central column, and distributed delays: right column). Figure 2 displays results for the heterogeneous networks (SW: top row, SF: bottom row) and the same delay distributions.

We observe a general trend towards synchronization when increasing the coupling strength and the average
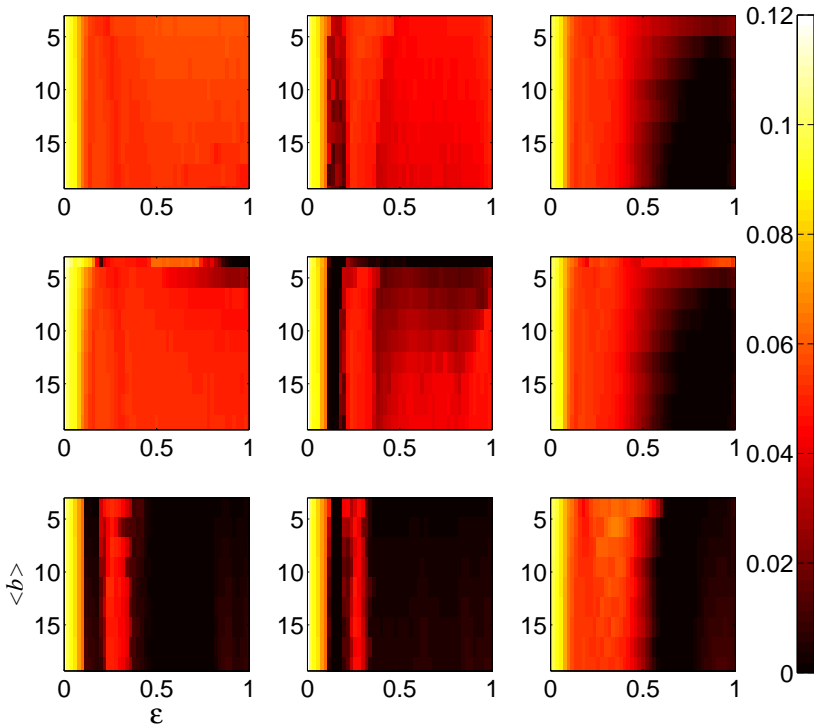

Fig. 1. (Color online) Plot of $\sigma^{2}$ in the parameter space (coupling strength, average number of neighbors) for three networks with regular topologies: nearest-neighbors (NN), top row, nearest-neighbors with central node (ST), middle row, and bottom row $K$-to-all (KA). The delays are: zero (left column), homogeneous $\left(\tau_{i j}=5 \forall i\right.$ and $j$, central column), and heterogeneous ( $\tau_{0}=5, c=2$, right column).
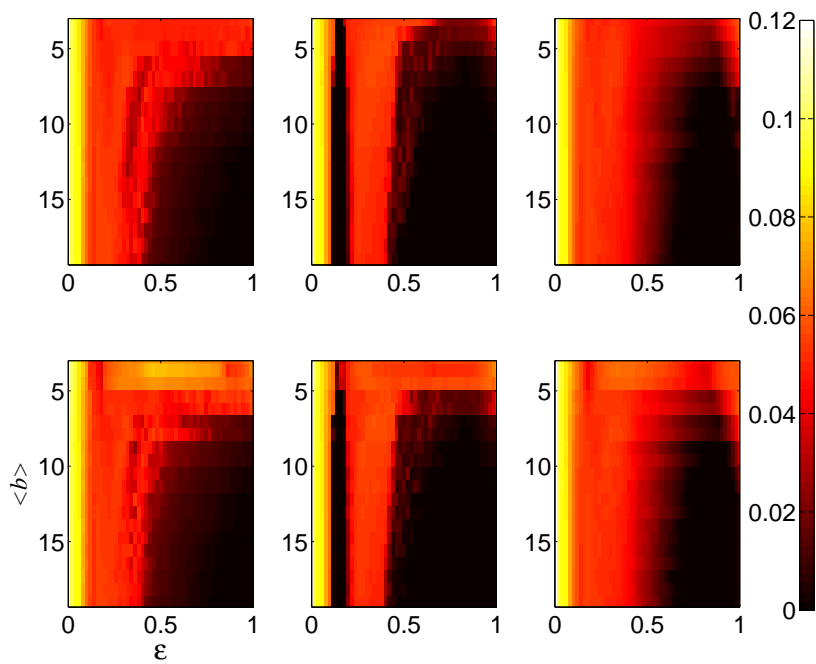

Fig. 2. (Color online) Plot of the order parameter $\sigma^{2}$, Eq 5 in the parameter space (coupling strength, average number of neighbors) for two heterogeneous networks of 200 nodes: SW (small-world, top row), SF (scale-free, bottom row). The delays are as in Fig. 1. no-delays (left column), homogeneous delays (center column), and heterogeneous delays (right column). Other parameters as Fig. 1. 
number of links. However we note some important differences depending on the topology and on the delays.

(i) Heterogeneous delays: if $\langle b\rangle$ is large enough, the synchronizability does not dependent on the topology, note the remarkable similarity of the five panels in the right columns of Figs. 1 and 2, for $\langle b\rangle \gtrsim 10$.

(ii) No-delays and homogeneous delays (left and central columns in Figs. (1) and 2): the topology makes a significant difference in regular networks (Fig. 11), and is less important in heterogenous networks (Fig. 22), although some differences can be observed for small $\langle b\rangle(\langle b\rangle \lesssim 10)$.

(iii) Regular networks: in Fig. 1 comparing the panels in the right and central columns we notice that the network KA (bottom row) is the one with better propensity to synchronization, while the network NN (top row) is the one exhibiting poorer synchronizability. We also notice that ST (middle row) has better synchronizability than NN when $\langle b\rangle$ is small, but there are no significant differences between them for large enough $\langle b\rangle$.

We note that for larger values of $\langle b\rangle$ (not shown in Fig. 1 because we focus on the weak connectivity region) the NN network eventually synchronizes. Synchronization occurs for $\epsilon$ above a certain value, $\epsilon^{*}$, that decreases with increasing $\langle b\rangle$, in good agreement with the results of Ref. 37. (see in particular Fig. 4(b) of [37, where the variable $\alpha$ plays the role of $\langle b\rangle$ here; increasing $\langle b\rangle$ resulting in a transition from local to global coupling).

(iv) Heterogeneous networks: in Fig. 2 we do not observe a significant difference: the panels in the top and bottom rows are similar, at least for $\langle b\rangle>5$.

(v) With homogeneous delays, middle column in Figs. 1 and 2 there is an "island of synchronization" in the region $\epsilon \sim 0.15$ to 0.19 . In spite of the fact that the coupling is weak, the network tends to synchronize, regardless of the topology. This is due to the fact that the delay times are even; for odd delays this synchronization window does not exist (see, e.g., Fig. 1 in 23 and Fig. 2 in 36]). In the middle column of Fig. 1 and 2 we observe that the "synchronization island" is robust and occurs for all the topologies.

\subsection{Characterization in terms of $\sigma^{\prime 2}$}

In order to investigate in more detail the two synchronization regimes (time-dependent and fixed-point synchronization) now we consider the synchronization indicator $\sigma^{\prime 2}$, Eq. (6), that tends to zero only when the network synchronizes in the fixed point. Figures 3 and 4 display colorcoded plots of $\sigma^{\prime 2}$ as a function of the coupling strength, $\epsilon$, and the average number of neighbors, $\langle b\rangle$, for the same delay distributions and network topologies as Figs. 2 and 3 respectively.

In the case of heterogenous delays, again we notice a remarkable similarity in the five panels in the right columns of Figs. 3 and 4 for $\langle b\rangle$ large enough, confirming that the synchronizability of the network is largely independent of its topology. However, we note that for the topology KA (bottom panel, right column in Fig. 3) the network losses synchrony if the coupling is too strong $(\epsilon \approx 1)$.
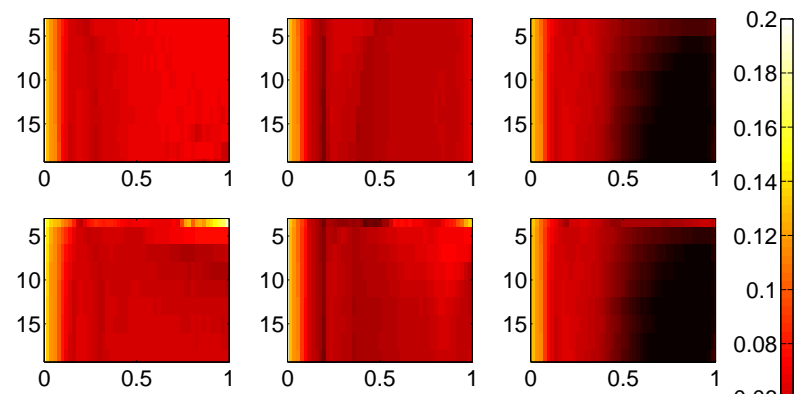

0.12
0.1
0.08
1
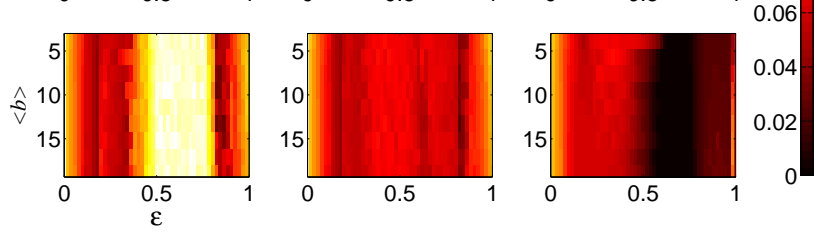

Fig. 3. (Color online) Plot of the order parameter $\sigma^{\prime 2}$, Eq. (6) in the parameter space (coupling strength, average number of neighbors) for three networks with regular topologies: nearestneighbors (NN), top row, nearest-neighbors with central node (ST), middle row, and nearest-neighbors with two central nodes (KA). Parameters are as in Fig. 1.

Regular networks: comparing the panels in the right and central columns of Fig. 3, we can notice that the network KA (bottom row) is the one with poorer synchronizability in the steady-state.

Heterogenous networks: in Fig. 4 is observed that the SW and SF topologies have very similar propensity for synchronization in the steady-state.

It can be noticed that, as the average number of links per node and the coupling strength increase, the networks with heterogenous delays tend to synchronize in the steady state, while the networks with no-delays or with homogenous delays do not [in Figs. 3 and 4 . compare the left and central columns with the right column]. This tendency is particularly clear in heterogeneous networks, Fig. 4. where in the left and in the central columns $\sigma^{\prime 2}$ is large for $\langle b\rangle \geq 15$ and $\epsilon \geq 0.8$ (right, bottom corner of the panels), while in the panels of the right column, $\sigma^{\prime 2}$ is zero or very small in that region.

The "synchronization island" discussed in the previous section is not observed in Figs. 3 and 4 because in this "island" the network synchronizes in a time-dependent state that has $\sigma^{\prime 2}$ different from zero.

\subsection{Connectivity threshold}

In the presence of heterogeneous delays, it can be expected that the average number of neighbors has to be above a certain value for the distribution of delays to play an effective role. This is indeed shown in Fig. [5, where we plot the synchronization indicators $\sigma^{2}$ and $\sigma^{\prime 2}$ in the plane $\left(c / \tau_{0}\right.$, $\langle b\rangle)$ for various network sizes and the SW topology. $\sigma^{2}$ and $\sigma^{\prime 2}$ are here plotted on a logarithmic scale to reveal the following features of the synchronization transition: 

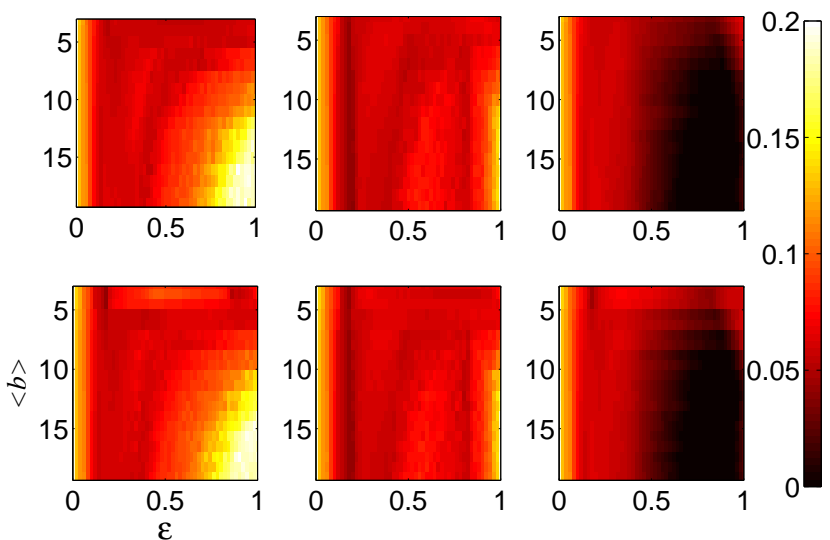

Fig. 4. (Color online) Plot of $\sigma^{\prime 2}$ in the parameter space (coupling strength, average number of neighbors) for three heterogeneous networks: SW (small-world, top row), RN (random network, middle row), SF (scale-free, bottom row); and the different delay distributions; instantaneous (left column), homogeneous delays (center column), and heterogeneous delays (right column). Parameters are as in Fig. 1.

for connectivity below $\langle b\rangle \approx 10$, the network does not synchronize, regardless of the network size and of the width of the delay distribution. Similar results are found for other values of $\epsilon$ and $\tau_{0}$. For $\langle b\rangle \geq 10$, as $c$ increases and the delays become more heterogeneously distributed there is a sharp transition to isochronous synchronization, reveled in the plot of $\sigma^{2}$ (left column), which shows a sharp boundary between the dark and light regions, at $c / \tau_{0}$ slightly below 0.5. The smooth transition seen in the plot of $\sigma^{\prime 2}$ (right column) reveals that initially the network does not synchronize in the fixed point but in a time-dependent state, and as $c$ increases and the delay distribution enlarges, it gradually approaches the fixed point.

\subsection{Influence of self-feedback links}

We assess the effect of self-feedback links by setting $\eta_{i i}=1$ $\forall i$, in the previously studied network topologies.

In a large enough network (as the one studied so far, with $N=200$ maps), the simulations show that feedback links have a small influence on the network synchronizability when the connectivity is low (when $\langle b\rangle \lesssim 0.5 N$ ), and their effect is negligible when $\langle b\rangle$ is larger. With $2 \mathrm{D}$ color-coded plots is difficult to appreciate the influence of the feedback links; therefore, we plot in Fig. 6 the global synchronization indicators vs. the coupling strength, for a fixed value of $\langle b\rangle$, that is as low as possible.

We consider three regular topologies: (i) a ring with two neighbors per node and a self-feedback link in each node (black solid line); (ii) the same ring without the selffeedback links (dot-dashed line, blue online); and (iii) a ST network composed by the ring with two neighbors per node (without self-feedback links) and a centrally connected node (dashed line, red online). It can be noticed that with no delays and with uniform delays (left and
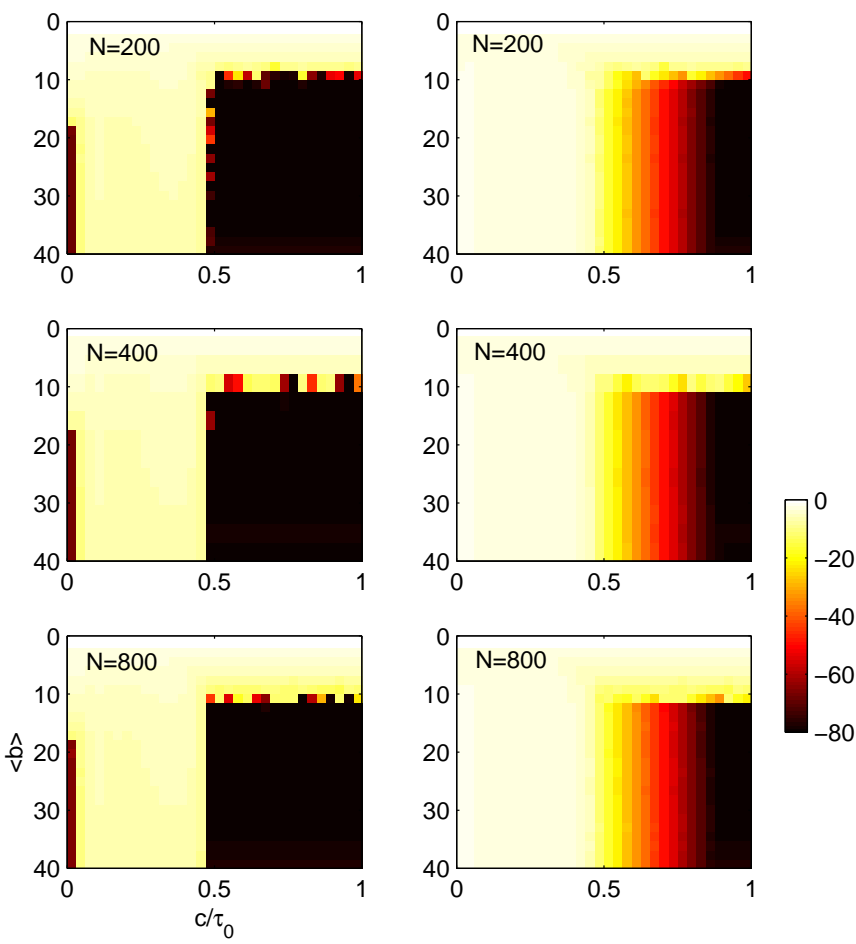

Fig. 5. (Color online) Plot of $\sigma^{2}$ (left column) and $\sigma^{\prime 2}$ (right column) in logarithmic scale, in the parameter space (normalized width of the delay distribution, $c / \tau_{0}$, average connectivity, $\langle b\rangle$ ) for three system sizes: $N=200$ (top row), $N=400$ (middle row), and $N=800$ (bottom row). Parameters are $\tau_{0}=5$ and $\epsilon=1$, SW topology.

central columns in Fig. 6), the ST network synchronizes for $\epsilon$ large enough, while the ring, with and without selffeedback links, does not. With randomly distributed delays (right column in Fig. [6), the three networks do not synchronize for any value of $\epsilon$.

Let us now consider the influence of feedback links in a smaller network of $N=20$ maps. Figure 6 displays the global synchronization indicator while Figs. 7-9 present some examples of the space and time evolution of the network. The figures are done by representing in a color scale the variable $x_{i}(t)$, with the space index $i$ on the horizontal axis and the time index $t$ on the vertical axis. The left column of Figs. 6-9 shows results for networks without feedback loops, and the right column, for the same networks with feedback loops. A relevant effect of the feedback can be observed on both, the global macroscopic indicator and on the microscopic network configuration. There are situations in which feedback loops enhance coherence, giving rise to spatially more ordered patterns (e.g., in Fig. 7, top and bottom row), while in others, on the contrary, feedback loops destroy the spatial coherence of the pattern (e.g., in Fig. 9). Interestingly, it can be observed that with random delays, Fig. 9, the synchronization is not always on a homogeneous state, but there are also static patterns with spatial "antiphase" arragement. The characterization of these patterns is in progress and will be reported elsewhere. 

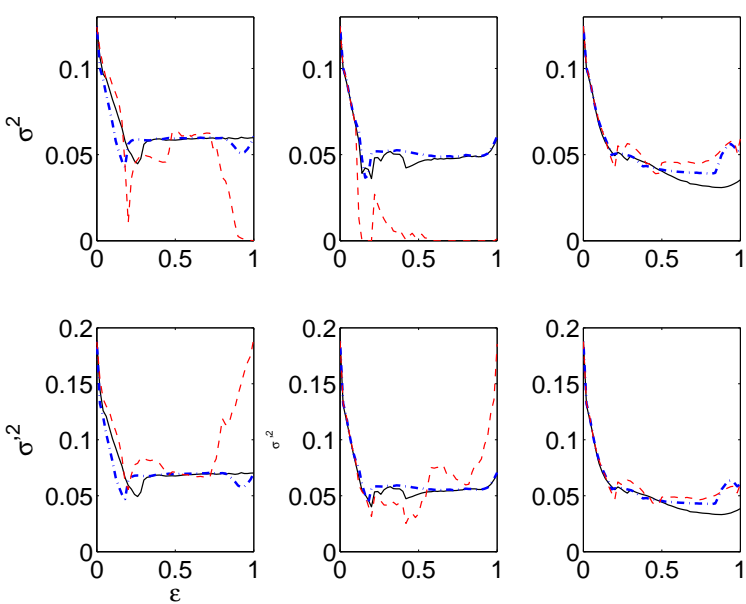

Fig. 6. (Color online) Plot of $\sigma^{2}$ (top row) and $\sigma^{\prime 2}$ (bottom row) vs. the coupling strength for a $\mathrm{NN}$ network with two neighbors per node and a feedback link (solid line), for the same ring without feedback links (dot-dashed line, blue online) and for the ST network composed by the ring plus a central node (dashed line, red online). The delays and other parameters are as in the previous figures: no-delays (left column), homogeneous delays (central column) and heterogeneous delays (right column).
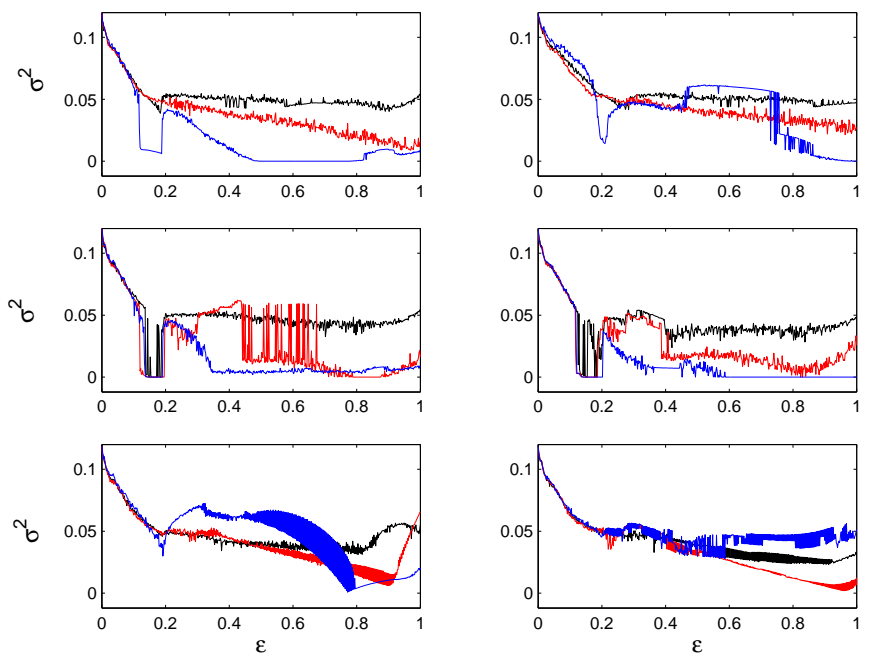

Fig. 7. (Color online) Synchronization indicator $\sigma^{2}$ for a small network of $N=21$ nodes without feedback loops (left column), and with feedback loops (right column). The delay distributions are: no delays (top row), fixed delays (middle row), and distributed delays (bottom row). The topologies are: a nearestneighbors ring (black), a ring with the addition of a central node (red) and a pure star network composed by a central hub connected to $N=20$ nodes (blue).
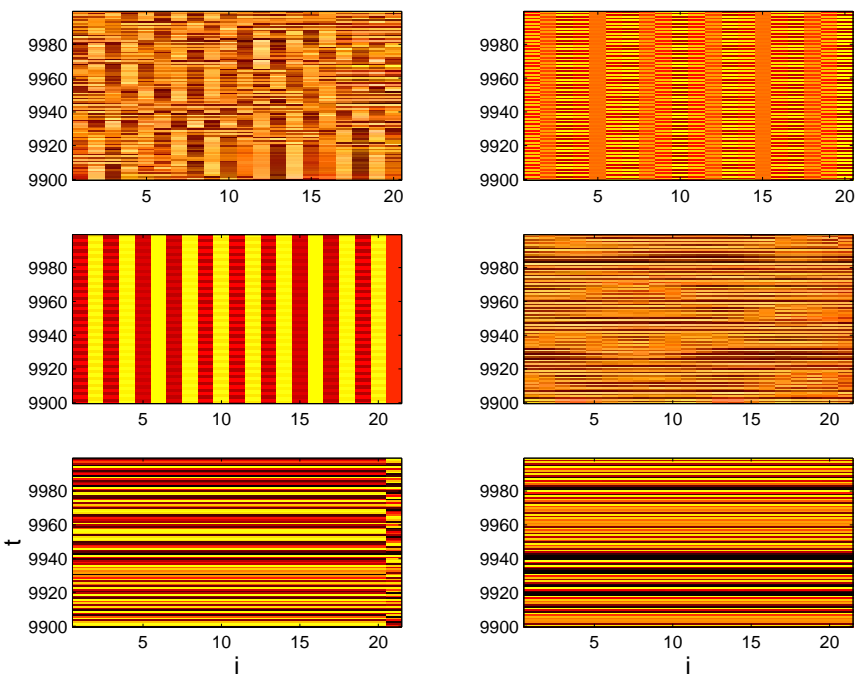

Fig. 8. (Color online) Synchronization patterns with instantaneous interactions. The network topologies are: a nearestneighbors ring of $N=20$ nodes (top row); a $N=20$ nearestneighbors ring with the addition of a central node (middle row) and a star network composed by a central hub connected to $N=20$ nodes (bottom row). Without feedback loops (left column), with feedback loops (right column). The coupling strength is $\epsilon=1$
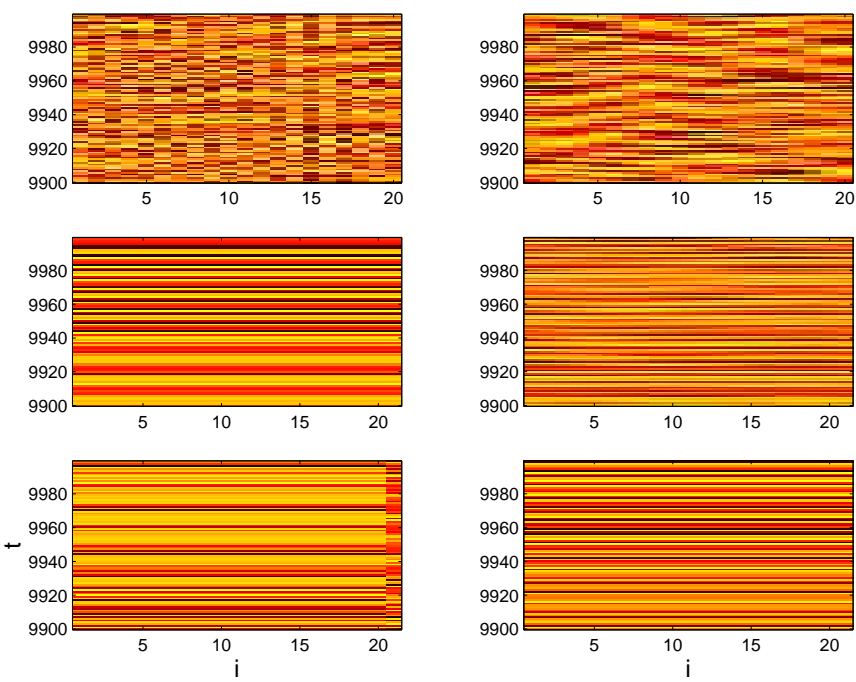

Fig. 9. (Color online) Synchronization patterns with homogeneous delays $\left(\tau_{0}=5, c=0\right)$. The network topologies are as in Fig. 7. (a),(b) $\epsilon=1$; (c)-(f) $\epsilon=0.8$. Without feedback loops (left column), with feedback loops (right column).

It is also interesting to notice that when the coupling strength is small (roughly speaking, when $\epsilon<0.2$ ) the dependence of the global synchronization indicators $\sigma^{2}$ and $\sigma^{\prime 2}$ with $\epsilon$, shown in Figs. 6 and 6, is very similar. This suggests that the global dynamics is almost independent of the topology, the connectivity, the network size and the delay distribution. We refer to this region as the "weak coupling" region. It has been recently shown that in this region all the nodes exhibit a qualitatively similar sym- 

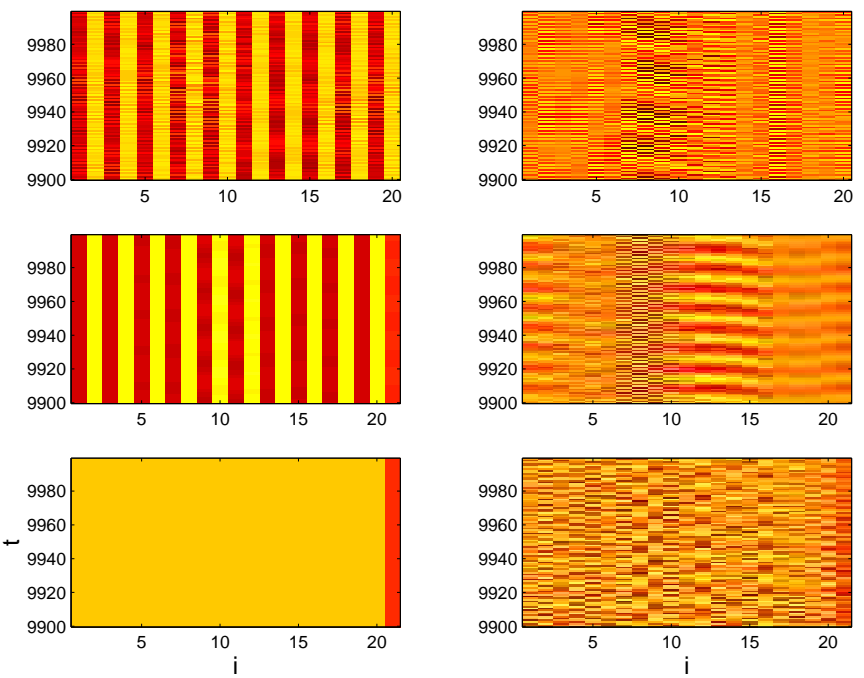

Fig. 10. (Color online) Synchronization patterns with heterogeneous delays $\left(\tau_{0}=5, c=2\right)$. The network topologies are as in Figs. 7 and 8. (a),(b) $\epsilon=0.9$; (c),(d) $\epsilon=1$; (e),(f) $\epsilon=0.8$. Without self-feedback loops (left column), with self-feedback loops (right column).

bolic dynamics, that, for instantaneous interactions, depends mainly on the network architecture and only to a small extent, on the local dynamics [40].

\section{Summary and Conclusion}

We studied the synchronizability of a network focusing on the roles of the connectivity, the topology, and the delay times that are associated with the links. The nodes were modeled by chaotic logistic maps, and various topologies and delay distributions were considered. For low connectivity (roughly speaking, when the mean number of links per node is $\langle b\rangle<0.1 N)$, and for weak coupling $(\epsilon<0.1)$, the network displays an irregular oscillatory dynamics, regardless of the network topology and the delay distribution. For large enough connectivity and coupling, the synchronization is mainly determined by the delay times: when the delays are homogeneously distributed the network shows collective synchronous time-dependent oscillations; when the delays are sufficiently distributed, the network synchronizes in a spatially homogeneous steadystate. The propensity towards these two synchronization regimes was characterized in terms of two indicators, $\sigma^{2}$ and $\sigma^{\prime 2}$ [Eqs. (5]) and (6)], the first one tends to zero when the network synchronizes isochronously $\left[x_{i}(t)=x_{j}(t) \forall\right.$ $i, j]$, while the second one tends to zero only when the network synchronizes in the steady state $\left[x_{i}(t)=x_{0} \forall i\right.$, with $x_{0}=f\left(x_{0}\right)$ being the fixed point of the uncoupled maps].

When the coupling strength is weak the dependence of the synchronization indicators with $\epsilon$ is very similar for all topologies and delay distributions considered. This suggest that in this region of "weak coupling" the network topology and the delay distribution play no relevant role in the dynamics. However, is important to remark that the global synchronization indicators employed, $\sigma^{2}$ and $\sigma^{\prime 2}$, have the limitation that they characterize only isochronal synchronization and steady-state synchronization respectively. In a complex network where the interactions among the units are not instantaneous, other synchronization patterns are also expected, such as states where the nodes are synchronized but with lag-times between them 38,39. An interesting indicator to analyze in future studies is the one that measures the average distance between the present state of a map and the delayed state of the maps interacting with it:

$$
\sigma^{2}=(1 / N) \sum_{i}\left(1 / b_{i}\right) \sum_{j} \eta_{i j}\left\langle\left(x_{i}(t)-x_{j}\left(t-\tau_{i j}\right)\right)^{2}\right\rangle_{t} .
$$

Also, it will be very interesting to analyze synchronization patterns using symbolic dynamics [40] and complexity indicators 41.42.

With heterogeneous delays we also found that there is a connectivity threshold below which the network does not synchronize, regardless of the network size. This minimum average number of neighbors is also independent of the delay distribution. Above the minimum connectivity level, as the width of the distribution increases, the plot of $\sigma^{2}$ reveals that there is a sharp transition to isochronous synchronization, while the plot of $\sigma^{\prime 2}$ reveals that the network does not synchronize in the fixed point but in a timedependent state, that gradually approaches the fixed point as the delays become more heterogeneous. Simulations were preformed for $S W$ topology; the study of how this connectivity threshold depends on the network topology is in progress and will be reported elsewhere.

We studied the influence of feedback loops in each node of the network; these feedback loops having the same delay distribution as the mutual interactions, and found that when the network is large, feedback loops have very little impact on the global synchronization indicators. However, they affect synchronizability of small networks, enhancing or degrading the synchronization, depending on the network architecture and on the delay distribution. As a future study it will be interesting to analyze the interplay of delayed feedback with instantaneous mutual coupling and vice-versa, instantaneous feedback with delayed coupling.

\section{Acknowledgments}

ACM and MPC acknowledge financial support from CSIC and PEDECIBA (Uruguay). CM acknowledges support from the "Ramon y Cajal" Program (Spain) and the European Commission (GABA project, FP6-NEST 043309).

\section{References}

1. S. H. Strogatz, "Exploring complex networks," Nature 410, 268-276 (2001).

2. See the special issue Pramana J. Phys. 70, 1 (2008): Proceedings of the Conference and Workshop on Perspectives in Nonlinear Dynamics 2007. 
3. J. M. Montoya, S. L. Pimm, and R. V. Sole, "Ecological networks and their fragility," Nature 442, 259-264 (2006).

4. D. Garlaschelli, G. Caldarelli, and L. Pietronero, "Universal scaling relations in food webs," Nature 423, 165-168 (2003).

5. H. Jeong, B. Tombor, R. Albert, Z. N. Oltvai, and A. L. Barabasi, "The large-scale organization of metabolic networks," Nature 407, 651-654 (2000).

6. N. Barkai and S. Leibler, "Robustness in simple biochemical networks," Nature 387 , 913-917 (1997).

7. C. S. Zhou, L. Zemanova, G. Zamora, et al. "Hierarchical organization unveiled by functional connectivity in complex brain networks," Phys. Rev. Lett. 97 , 238103 (2006).

8. V. M. Eguiluz, D. R. Chialvo, G. A. Cecchi, M. Baliki, and A. V. Apkarian, "Scale-free brain functional networks," Phys. Rev. Lett. 94, 018102 (2005).

9. M. Kuperman and G. Abramson, "Small world effect in an epidemiological model," Phys. Rev. Lett. 86, 2909-2912 (2001).

10. S. Eubank, H. Guclu, V. S. A. Kumar, M. V. Marathe, A. Srinivasan, Z. Toroczkai, and N. Wang, "Modelling disease outbreaks in realistic urban social networks," Nature 429, 180-184 (2004).

11. R. Albert, H. Jeong, and A. L. Barabasi, "Error and attack tolerance of complex networks," Nature 406, 378-382 (2000).

12. G. Palla, I. Derenyi, I. Farkas, T. Vicsek, "Uncovering the overlapping community structure of complex networks in nature and society," Nature 435, 814-818 (2005).

13. J. M. Kumpula, J. P. Onnela, J. Saramaki, et al. "Emergence of communities in weighted networks," Phys. Rev. Lett. 99 , 228701 (2007).

14. J. M. Buldu, P. Cano, M. Koppenberger, J. A. Almendral, and S. Boccaletti, "The complex network of musical tastes," New J. Phys. 9, 172 (2007).

15. N. J. Guido, X. Wang, D. Adalsteinsson, D. McMillen, J. Hasty, C. R. Cantor, T. C. Elston, J. J. Collins, "A bottom-up approach to gene regulation," Nature 439, 856860 (2006).

16. G. M. Suel, J. Garcia-Ojalvo, L. M. Liberman, M. B. Elowitz, "An excitable gene regulatory circuit induces transient cellular differentiation," Nature 440, 545-550 (2006).

17. R. Guimera, L. A. N. Amaral, "Functional cartography of complex metabolic networks," Nature 433, 895 (2005).

18. S. C. Manrubia, A. S. Mikhailov, D. H. Zanette, "Emergence of Dynamical Order. Synchronization Phenomena in Complex Systems", (World Scientific, Singapore, 2004).

19. A. E. Motter, C. Zhou, and J. Kurths, "Network synchronization, diffusion, and the paradox of heterogeneity," Phys. Rev. E 71, 016116 (2005).

20. M. Chavez, D. U. Hwang, A. Amann, H. G. E. Hentschel, S. Boccaletti, "Synchronization is enhanced in weighted complex networks," Phys. Rev. Lett. 94, 218701 (2005).

21. C. S. Zhou and J. Kurths, "Dynamical weights and enhanced synchronization in adaptive complex networks," Phys. Rev. Lett. 96, 164102 (2006).

22. T. Nishikawa, A. E. Motter, Y. C. Lai, et al., "Heterogeneity in oscillator networks: Are smaller worlds easier to synchronize?," Phys. Rev. Lett. 91, 014101 (2003).

23. F. M. Atay, J. Jost and A. Wende, "Delays, Connection Topology, and Synchronization of Coupled Chaotic Maps," Phys. Rev. Lett. 92, 144101 (2004).
24. C. Masoller and A. C. Martí, "Random Delays and the Synchronization of Chaotic Maps," Phys. Rev. Lett. 94, 134102 (2005).

25. A. C. Martí, M. Ponce and C. Masoller, "Chaotic maps coupled with random delays: connectivity, topology, and network propensity for synchronization," Physica A, 371, 104 (2006).

26. M. E. J. Newman and D. J. Watts, "Scaling and percolation in the small-world network model," Phys. Rev. E 60, 7332 (1999); "Renormalization group analysis of the small-world network model," Phys. Lett. A 263, 341-346 (1999).

27. R. Albert and A-L. Barabási, "Statistical Mechanics of Complex Networks," Rev. Mod. Phys. 74, 47 (2002).

28. M. G. Rosenblum and A. G. Pikovsky, "Controlling synchronization in an ensemble of globally coupled oscillators", Phys. Rev. Lett. 92, 114102 (2004).

29. O. V. Popovych, V. Krachkovskyi, P. A. Tass, "Twofold impact of delayed feedback on coupled oscillators", Int. J. Bif. Chaos 17, 2517 (2007).

30. E. Klein, N. Gross, M. Rosenbluh, W. Kinzel, L. Khaykovich and I. Kanter, "Stable isochronal synchronization of mutually coupled chaotic lasers", Phys. Rev. E 76, 066214 (2006).

31. I. B. Schwartz and L. B. Shaw, "Isochronal synchronization of delay-coupled systems", Phys. Rev. E 75, 046207 (2007).

32. T. Omi and S. Shinomoto, "Can distributed delays perfectly stabilize dynamical networks?", Phys. Rev. E 77, 046214 (2008).

33. R. Morgado, M. Ciesla, L. Longa and F. A. Oliveira, "Synchronization in the presence of memory", Europhys. Lett. 79, 10002 (2007).

34. S. Kim, S. H. Park and C. S. Ryu, "Multistability in coupled oscillator systems with time delay", Phys. Rev. Lett. 79, 2911 (1997).

35. A. Roxin, N. Brunel and D. Hansel, "Role of delays in shaping spatiotemporal dynamics of neuronal activity in large networks", Phys. Rev. Lett. 94, 238103 (2005).

36. P. G. Lind, A. Nunes, and J. A. C. Gallas, "Impact of bistability in the synchronization of chaotic maps with delayed coupling and complex topologies", Physica A 371, 100 (2006).

37. R.L. Viana, C. Grebogi, S.E. de S. Pinto, S.R. Lopes, A.M. Batista, and J. Kurths, "Bubbling bifurcation: Loss of synchronization and shadowing breakdown in complex systems", Physica D 20694 (2005).

38. C. Masoller, A. C. Martí and D. H. Zanette, "Synchronization in an array of globally coupled maps with delayed interactions", Physica A 325, 186 (2003).

39. C. M. Gonzalez, C. Masoller, C. Torrent and J. GarciaOjalvo, "Synchronization via clustering in a small delaycoupled laser network", Europhys. Lett. 79, 64003 (2007).

40. S. Jalan, J. Jost and F. M. Atay, "Symbolic synchronization and the detection of global properties of coupled dynamics from local information", Chaos 16, 033124 (2006).

41. J. R. Sanchez and R. Lopez-Ruiz, "A method to discern complexity in two-dimensional patterns generated by coupled map lattices", Physica A 355633 (2005).

42. O. A. Rosso, H. A. Larrondo, M. T. Martin, A. Plastino, and M. A. Fuentes, "Distinguishing noise from chaos", Phys. Rev. Lett. 99154102 (2007). 